\title{
Location Design of Electric Vehicle Charging Facilities: A Path-Distance Constrained Stochastic User Equilibrium Approach
}

\author{
Wentao Jing, ${ }^{1}$ Kun An, ${ }^{1}$ Mohsen Ramezani, ${ }^{2}$ and Inhi Kim ${ }^{1}$ \\ ${ }^{1}$ Institute of Transport Studies, Department of Civil Engineering, Monash University, 23 College Walk, Melbourne, VIC, Australia \\ ${ }^{2}$ School of Civil Engineering, The University of Sydney, Sydney, NSW, Australia \\ Correspondence should be addressed to Inhi Kim; inhi.kim@monash.edu
}

Received 8 May 2017; Revised 3 September 2017; Accepted 13 September 2017; Published 16 October 2017

Academic Editor: Chi Xie

Copyright (C) 2017 Wentao Jing et al. This is an open access article distributed under the Creative Commons Attribution License, which permits unrestricted use, distribution, and reproduction in any medium, provided the original work is properly cited.

\begin{abstract}
Location of public charging stations, range limit, and long battery-charging time inevitably affect drivers' path choice behavior and equilibrium flows of battery electric vehicles (BEVs) in a transportation network. This study investigates the effect of the location of BEVs public charging facilities on a network with mixed conventional gasoline vehicles (GVs) and BEVs. These two types of vehicles are distinguished from each other in terms of travel cost composition and distance limit. A bilevel model is developed to address this problem. In the upper level, the objective is to maximize coverage of BEV flows by locating a given number of charging stations on road segments considering budget constraints. A mixed-integer nonlinear program is proposed to formulate this model. A simple equilibrium-based heuristic algorithm is developed to obtain the solution. Finally, two numerical tests are presented to demonstrate applicability of the proposed model and feasibility and effectiveness of the solution algorithm. The results demonstrate that the equilibrium traffic flows are affected by charging speed, range limit, and charging facilities' utility and that $\mathrm{BEV}$ drivers incline to choose the route with charging stations and less charging time.
\end{abstract}

\section{Introduction}

Carbon-based emissions and greenhouse gases are critical global issues, where transport sector is a significant contributor. A cost-effective strategy for reducing emissions is efficient use of alternative fuels. Cities, businesses, and governments have recognized electric vehicles (EVs) as an indispensable part of smart and sustainable city frameworks [1], because, comparing to conventional internal combustion engines, EVs are more energy efficient [2]. Moreover, battery electric vehicles (BEVs), as a type of alternative fuel vehicles, have been developed as a promising solution for reducing local air pollution at the point of operation [3], greenhouse gas emissions [4], dependency on fossil oil, and improving energy safety. Furthermore, EVs can be utilized to store energy from renewable resources, such as wind, wave power, and solar cells, to smoothen out the daily power fluctuation in low peak periods [5] with the development of vehicle-togrid (V2G) technology [6-8]. For consumers, the monetary savings of switching to a BEV can be significant due to cheaper electricity cost comparing with gasoline [9]. However, the early BEV users still suffer from the inconvenience of limited driving range, long charging time, and insufficient public charging stations $[1,10]$.

Currently the driving range of EVs can vary greatly between $60 \mathrm{~km}$ and $400 \mathrm{~km}$ by model and manufacturer, while most of them have ranges between $100 \mathrm{~km}$ and $160 \mathrm{~km}$ [11]. The EVs can be recharged using plug-in charging or battery-swapping facilities. The plug-in charging is categorized by voltage and power levels, leading to different charging times. Slow charging usually takes hours to charge while fast charging can achieve 50\% charge in 10-15 minutes [11]. Range anxiety, when the driver is concerned that the vehicle will run out of battery before reaching the destination, is a major hindrance for the market penetration of EVs [12] and will inevitably add a certain level of restrictions to BEV drivers' path choices, at least in a long future period prior to the massive coverage of recharging infrastructures [13]. 
Governments and automotive manufacturers have recognized the environmental value of EVs and, therefore, are encouraging BEV ownership through economic incentives and more public charging station deployment [14].

Explicitly incorporating the range limit into facility location problem (FLP) can be traced back to flow refueling facility location problems (FRFLP) which utilized optimization models to determine a set of locations to serve the refueling demand in a network subject to a financial budget. One branch of FRFLP sought to maximize demand coverage by locating a fixed number of refueling facilities, which was referred to as the maximal covering location problem (MCLP). This problem has been typically formulated as flow refueling location model (FRLM) [15-18], which served demand along their shortest paths rather than demand at their end points to maximize the coverage of these flows. Typically, they used modifications of flow-capturing or flow interception location models (FILM) [19, 20], which were path-based version of MCLP. In FILM, for each O-D pair, the shortest path between the O-D pair is considered as covered if it passes through at least one node that contains a refueling facility. The developed FRLM models have been compared empirically for specific scenarios in order to choose one location model over another [21]. Furthermore, in another attempt, a flow-based refueling-station-location model was proposed based on a set covering concept and vehicle-routing logics considering both intercity and intracity travel [22, 23]. The above model was reformulated and a flexible mixedinteger linear programming model was presented, which was able to obtain an optimal solution much faster than the previous set cover version. Moreover, the model also could be solved in the maximum cover form [24].

Along another track, a large variety of other approaches have been proposed to address the locations of EV public charging infrastructures. Huang et al. [11] proposed a geometric segmentation method to find the optimal location for both slow and fast charging stations. Sweda and Klabjan [25] developed an agent-based decision support system and a variant maximal covering location problem for EV charging infrastructure deployment. Asamer et al. [26], by using 800 electric taxis' operational data in the city of Vienna, Austria, proposed a two-phase decision support system. Nie and Ghamami [3] presented a conceptual optimization model to analyze travel by EV along a long corridor whose objective was to select the battery size and charging capacity (in terms of both the charging power at each station and the number of stations needed along the corridor) to meet a given level of service. They further proposed a fixed charge facility location model with charging capacity constraints, considering drivers' preference for familiar parking lots [27]. Chen et al. [28] investigated the optimal deployment of charging stations and lanes along a long traffic corridor to serve the charging need of EVs and examined the competitiveness of charging lanes over charging stations. Xi et al. [29] developed a simulationoptimization model that determined where to locate EV charging stations to maximize their use by privately owned EVs. Jung et al. [30] reported a simulation-optimization location model including an upper level multiple-server allocation model with queueing delay and a lower level dispatch simulation and provided a solution algorithm that featured itinerary-interception, stochastic demand, and queueing delay. Dong et al. [31] analyzed the impact of public charging station deployment on increasing electric miles traveled. By considering transportation and power networks and maximizing the social welfare, He et al. [32] developed an equilibrium-based modeling framework for locating plug-in charging facilities. Riemann et al. [33] incorporated stochastic user equilibrium (SUE) into a FCLM and aimed to capturing the maximum EV path flow on a network. A global optimal solution was applied to solve the proposed model. Wu and Sioshansi [34] proposed a stochastic flow-capturing model to optimize the location of fast charging stations, addressing the uncertainty of BEV flows. Zhu et al. [35] proposed a model that simultaneously handled the problem of where to locate the charging stations and how many chargers should be established in each charging station to minimize the total cost.

The location design problem of charging facilities can be modeled as a Leader-Follower Stackelberg game where the decision makers are the leaders who decide the facility deployment and the BEV users are the followers who can choose their paths freely. Most of the previous studies focused on user equilibrium (UE) problems with BEVs. Among these studies, Jiang et al. [13] first introduced a path-constrained deterministic traffic assignment problem and further extended this work by considering trip chain and range anxiety analysis [36-39]. Zheng et al. [40] presented a bilevel model to locate charging facility and minimize all users cost in the upper level and to find path-constrained equilibrium $\mathrm{BEV}$ flows in the lower level. Jing et al. [41] provided a comprehensive review for the equilibrium network modeling. However, the driving distance limit, to the best of our knowledge, has not been considered in stochastic network equilibrium models, especially in the mixed flow transport network. Moreover, to tackle the range anxiety problem with a limited budget, the charging facilities should be accessible to as many EVs as possible [11]. It can be an efficient way to deploy the public charging facilities on the links where most BEV drivers use to increase the utilization and perception of the public charging facilities, which promotes BEV acceptance and relieve range anxiety [31]. Given the high cost of building public charging stations and financial constraints, it is essential to optimize the location of facilities in a network that provides the maximum exposure and utilization by BEV drivers. Since various factors influence BEV drivers' charging decision, such as stochasticity of range anxiety, initial battery energy state, battery energy consumption ratio, and battery capacity, considering those factors in the model is of great importance.

In this study, we present a novel bilevel public charging infrastructure location model that maximizes the total captured BEV link flows, considering BEV range limits and SUE principle to capture BEV drivers' route choice behavior in a network with mixed BEV and gasoline vehicles (GVs). The objective of the upper level of the model is to cover the maximum BEV link flows in a network by deploying a given number of charging facilities. In other words, the model aims to maximize the number of BEVs who can access the charging facilities along their routes. In the lower level, the stochastic traffic assignment on the network is the primary factor that 
determines the location of charging facility deployment. In general, a network equilibrium problem with multiple vehicle/mode classes cannot be written as a convex mathematical programming model, due to the existence of the asymmetric Jacobi matrix caused by different impacts on travel cost from different vehicle/mode classes [42]. The approaches to deal with the asymmetric Jacobian elements can be attributed to Jiang and Xie [43] and Ryu et al. [44]. It should be noted that relaxing the asymmetric restriction inevitably degrades the realism of traffic assignment model. However, in our model, the general compositions of path travel cost functions of the two vehicle classes, that is, GVs and BEVs, are similar. The only differences between these two types of vehicles lie in two flow-independent terms, namely, charging facility utility and charging time and thus their flow-time impacts on each other are symmetric (i.e., the impact of GVs on the travel times of BEVs is the same as the impact of BEVs on the travel times of GVs).

Modeling a traffic network with realistic refueling behaviors may require accommodating different routing objectives (e.g., minimization of travel time, charging time, and/or fuel consumption), different refueling services (e.g., batterycharging service or battery-swapping service), and different types of vehicles (e.g., GVs and BEVs) [38]. All these factors result in different path travel cost perception and route choice behaviors. It is evident that BEV drivers have inherent differences in travel behavior from GV drivers and specifically range limit, charging speed, and charging stations locations have significant influence on BEV drivers' decision-making process [45].

This paper focuses on several factors to explicitly capture BEV drivers' behavior with the stochastic traffic assignment. However, we understand the limitations of the stochastic traffic assignment in the lower level for accurately capturing realistic situations. It is believed that the results from this paper can provide some guidelines for locating BEV charging facility and basic insights of BEV drivers' behavior. Despite all the realistic situations, most data, such as demand, initial battery state of charge, and actual range limit, are difficult to obtain and this method and objective are easy to implement especially at the early stage of expanding EV market share. First, BEVs' range limit is considered as travel distance such that any path whose distance is greater than its range limit (referred to as infeasible paths) would not be chosen if the existing charging facility could not help finish the trip. Second, availability of charging facility would affect the route choice in a way that those infeasible paths may become feasible after recharging at the charging facilities on the path. Furthermore, the utility theory is applied to charging facility; that is, BEV drivers are more likely to choose the path with charging facilities over others without charging facilities even if they have equal path travel time. In addition, traffic congestion effects on travel time are also taken into consideration in BEV drivers' route choice behavior but not in the range limit constraint. Lastly, under the principle of perceived individual cost minimization, the path cost structure in the lower level model consists of flow-dependent path travel time, charging time, and utility of charging facilities (equivalent to given amount of travel time reduction). Specifically, the lower level model can be stated as follows: in a traffic network with fixed GV and BEV travel demand between each O-D pair and a set of charging facilities at known locations, the problem is to find such a traffic flow pattern that each trip maker chooses a path along which his or her least perceived cost is minimized and the vehicle can be charged before running out of energy before arriving at the destination. Meanwhile, no one can improve his/her perceived travel cost by unilaterally changing a path. Given the sufficient coverage of gasoline stations and GVs' large fuel capacity, GVs' route choice is not affected by any other costs incurred by refueling requirement, except for travel time.

The contributions of this study are threefold. Firstly, a maximal flow-covering (MFC) model, that is, a modification of classic MCLP, is proposed to maximize BEV flow coverage by locating a fixed number of charging facilities in the bilevel, equilibrium-optimization framework. Coverage is achieved when the charging facilities are located on the BEV route. Secondly, the effects of driving distance limit constraints, charging facility availability, charging facility utility, and traffic congestion are accommodated in BEVs' route choice behavior. The equilibrium BEV flow pattern is determined endogenously by the general SUE traffic assignment model with driving distance limit constraints, in which the mutual interactions between the location of charging facilities and resultant equilibrium BEV link flow patterns are modeled. Finally a heuristic algorithm is proposed to solve the mixedinteger nonlinear program.

The remainder of this paper is organized as follows. In Sections 2 and 3, we elaborate the problem definition and formulation. Section 4 presents the solution methodology and details its algorithmic implementations, while Section 5 describes the numerical results from applying the algorithmic procedure for a small network and Sioux Falls network. In the end, we conclude the article and point out some future research directions in Section 6.

\section{Problem Description, Assumptions, and Notation}

BEVs rely entirely on electricity as a single power source and are designed to be charged at the charging facilities. BEVs' electricity consumption is typically proportional to the driving distance, resulting in a driving range limit because of the battery capacity. On the basis of current battery technology, charging a BEV still takes more time than refueling a GV's fuel tank. The distance limit, the charging time, and the location of the charging facilities inevitably change BEV drivers' route choice behavior in a stochastic manner where BEV drivers may have imperfect information regarding their travel cost over the entire mixed flow (i.e., BEVs and GVs) traffic network. The massive adoption of BEVs requires a certain level of coverage of the charging facility. Given the financial budget and high cost of installing public chargers, it is a sound approach to maximize the passing BEV population on the links where charging facilities are deployed.

This paper considers a strongly connected transportation network with both BEVs and GVs demands, denoted by 
$G=(N, A)$, where $N$ is the set of nodes and $A$ is the set of links. $R \subseteq N$ and $S \subseteq N$ denote the sets of origins and destinations, respectively. The objective of this proposed bilevel model is to locate a given number of $\mathrm{BEV}$ charging facilities for covering maximum BEV flows on the mixed traffic flow network. All the candidate charging facility locations are grouped into a set of pseudonodes in the middle of the links denoted by $Z$. GVs and BEVs are distinguished by their driving distance limits, travel cost composition, and the availability of refueling facilities.

Without loss of generality, the following assumptions are made:

(A1) The technological characteristics of BEVs and demographic features of BEV drivers are homogeneous in the network, and so are GVs and GV drivers. Only one type of $\mathrm{BEV}$ with identical driving distance limit and battery consumption rate is considered.

(A2) Every vehicle is fully charged at its origin.

(A3) The variation of BEV drivers' range anxiety level and risk-taking behaviors are ignored.

(A4) A charging facility is deployed on the midpoint of the link in the network.

(A5) The facilities have unlimited charging capacity. Hence, an EV can get charged without delay after its new arrival. En route charging time at the public charging facilities is linear related to the remaining distance to reach the destination.

(A6) The BEV link flow is covered if a charging facility exists on this link.

(A7) The deployment of a charging facility on a route/path would increase the "attractiveness" or "utility" of this route. The utility of a charging facility is considered as a fixed value and converted into travel time reduction.

(A8) Travel demand of both GV and BEV between each O$D$ pair is fixed. That is, elastic and stochastic demands are not considered in this model.

See the Notations for variables and parameters used throughout this paper, where subscripts $g$ and $e$ indicate variables or parameters associated with GV and BEV, respectively.

\section{Model Formulation}

In this section, we formulate the bilevel optimization model for the charging facility location problem. Bilevel problems split the decisions of the system planner (leader, i.e., infrastructure developer in this paper) and system users (followers, i.e., drivers) into two levels so that the subproblems are solvable and an iterative approach can be used to achieve an equilibrium state. The upper level aims at determining the locations of charging facilities to increase an objective to maximize the covered BEVs flows assuming BEVs flows remain unchanged. The lower level subproblem is characterized as BEV drivers' route choice behavior with a generalized travel cost structure. SUE conditions with mixed BEVs and GVs assuming fixed locations of charging facilities from the upper level subproblem are analysed.
3.1. Preliminaries. A feasible path for GVs between a given O-D pair may be infeasible for BEVs because of the limited driving distance range and absence of a charging facility. Hence, a feasible path used by GVs can be decomposed into several parts for BEVs according to whether a charging action should be taken by BEV drivers at each charging station. To model BEVs paths, three notions, namely, subpath, pure subpath, and feasible subpath, proposed by Xie and Jiang [38], are introduced in the formulation of the lower level stochastic assignment problem and three charging action based scenarios are analyzed as follows.

Subpath. A part of path $k$ connecting O-D pair $(r, s)$ is a subpath if charging stations are located at the head and tail nodes/pseudonodes of this part. A subpath consists of a number of consecutive links and half links since we assume charging stations locate in the middle of the links. We denote $k^{i j}, i, j \in Z$, as a subpath of path $k$, where charging station $i(j)$ is the head (tail) node of this subpath. $l_{k}^{r s, i j}$ is the length of the subpath.

Pure Subpath. Subpath $k^{i j}$ is a pure subpath if there are no other charging facilities on this subpath except $i$ and $j$.

Feasible Subpath. Subpath $k^{i j}$ is feasible on path $k$ of O-D pair $(r, s)$, if its length is no greater than BEV driving distance limit; that is, $l_{k}^{r s, i j} \leq D_{e}$.

The concept of subpaths allows us to better illustrate the $\mathrm{BEV}$ drivers' path travel cost structure and add the driving distance constraint.

The generalized path travel cost is composed of three parts: path travel time, path charging time, and equivalent travel time reduction (the utility of charging facilities on attracting BEV drivers). Without loss of generality, we consider 3 scenarios based on the relationship between the driving distance limit $D_{e}$ and subpath distances. For a given path $k$ shown in Figure 1, path travel time and equivalent travel time reduction are fixed and can be represented by a consistent form: $c_{k}^{r s}+t_{u, k}^{r s}$, where $t_{u, k}^{r s}=2 \cdot t_{u}^{o}$. Note that $t_{u}^{o}$ is a nonpositive value.

Scenario 1. There is no need for charging. When $l_{k}^{r s} \leq D_{e}$, the BEV driver can reach the destination without en route charging. The generalized path travel cost is $\bar{c}_{k e}^{r s}=c_{k}^{r s}+2 \cdot t_{u}^{o}$.

Scenario 2. If any pure subpath distance exceeds the driving distance limit $D_{e}$, this path becomes infeasible to BEV drivers. In other words, if path $k$ cannot be decomposed into a set of feasible subpaths, path $k$ is not feasible. In this case, the generalized path travel cost becomes extremely large and the probability of choosing this path is zero.

Scenario 3. Charging is needed to reach the destination. If the path distance is larger than the distance limit (i.e., $l_{k}^{r s, r s} \geq D_{e}$ ) and the distances of its all pure subpaths are less than $D_{e}$, the BEVs need to charge at least once. BEV s would charge as little as possible to reduce the path travel time. The minimum charging time is $t_{c, k}^{r s}=\varepsilon \cdot\left(l_{k}^{r s, r s}-D_{e}\right)$. The generalized path travel cost is $\bar{c}_{k e}^{r s}=c_{k}^{r s}+2 \cdot t_{u}^{o}+t_{c, k}^{r s}$. 


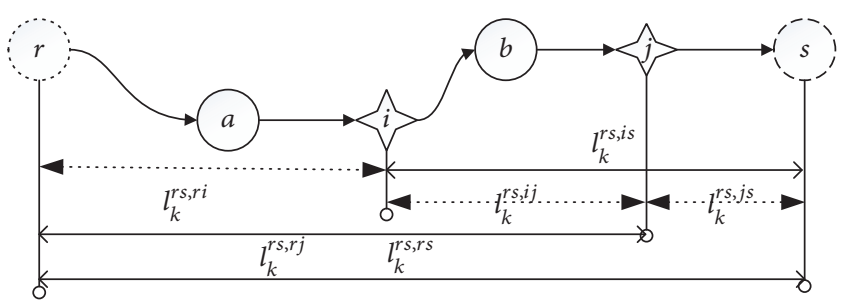

$\diamond$ Charging facility

Origin

(i) Destination

FIgURE 1: Illustration of subpaths definitions. We consider a path $k$ of O-D pair $(r, s)$, along which nodes $i$ and $j$ are located in the middle of links $a b, b s$, respectively. There exist 3 pure subpaths denoted by dotted lines, namely, $k^{r i}, k^{i j}$, and $k^{j s}$, and another 3 subpaths $k^{r j}, k^{r s}$, and $k^{i s}$ by solid lines on path $k$. These subpaths are feasible if their distance is less than the BEV driving distance limit $D_{e}$.

For GVs, the generalized path travel cost is $\bar{c}_{k g}^{r s}=c_{k}^{r s}$. Hence, BEV drivers are more likely to choose path $k$ than GV users under Scenario 1 due to the utility (attractiveness) of the charging facilities on this path, while only GV drivers would choose this path under Scenario 2 because of the infeasible pure subpath. A trade-off between charging time and charging facility utility should be made to identify the generalized travel cost difference of BEVs and GVs under Scenario 3. For example, charging time of fast charging or battery swapping may be shorter than the equivalent travel time reduction converted from the charging facility utility, and thus more BEVs would be assigned to this path even if they may need several charging instances on this path. If multiple charging stations are available on a path, BEV drivers will go through the following process to decide whether charging should be conducted at a station. Let us consider Scenario 3 only where each pure subpath is feasible for BEVs to reach the destination without running out of energy. When arrived at a charging station, BEVs would not charge at the current charging station if they can reach the next one without charging.

3.2. Bilevel Model Formulation. Given the key concepts and terms above, we define the upper level problem as

$$
\begin{array}{rl}
\max _{\mathbf{x}} & F[\mathbf{x}, \mathbf{v}(\mathbf{x})] \\
\text { Subject to } & E[\mathbf{x}, \mathbf{v}(\mathbf{x})] \leq 0,
\end{array}
$$

where $\mathbf{v}(\mathbf{x})$ is implicitly determined in the lower level problem

$$
\begin{array}{cl}
\min _{\mathbf{v}} & f[\mathbf{x}, \mathbf{v}] \\
\text { Subject to } & e[\mathbf{x}, \mathbf{v}] \leq 0,
\end{array}
$$

where $F$ and $E$ are the objective function and constraints of the upper level problem while $f$ and $e$ are those of the lower level distance-constrained SUE model. $F$ models the total covered BEV link flows and $E$ guarantees the number of charging facilities to be equal to the given design value. $\mathbf{x}$ and $\mathbf{v}$ are decision variables for upper and lower level problems; that is, $\mathbf{x}$ and $\mathbf{v}$ denote charging facility locations and BEV link flow pattern, respectively. Subsequent sections detail the mathematical properties of both upper and lower level subproblems.

Furthermore, in the lower level distance-constrained SUE problem in mixed traffic flow networks, the link performance functions are assumed to be a BPR (Bureau of Public Road) type function as follows:

$$
t_{a}\left(v_{a, g}, v_{a, e}\right)=t_{a}^{0}\left(1+0.15 \times\left(\frac{v_{a, g}+v_{a, e}}{H_{a}}\right)^{4}\right),
$$

$a \in A$.

As $t_{c, k}^{r s}$ and $t_{u}^{0}$ are flow-independent, we can easily obtain the Jacobi matrix for the lower level problem, with its elements given for GVs and BEVs, respectively, as follows:

$$
\frac{\partial \bar{c}_{k g}^{r s}}{\partial v_{a, e}}=\frac{\partial \bar{c}_{k e}^{r s}}{\partial v_{a, g}}=0.6 \sum_{a \in A} t_{a}^{0} \delta_{a, k}^{r s} \frac{\left(v_{a, g}+v_{a, e}\right)^{3}}{H_{a}{ }^{3}} .
$$

This proves that the Jacobi matrix is symmetric so that the lower level model can be established as a convex mathematical problem.

3.2.1. Upper Level Formulation. The upper level problem aims to maximize the total covered BEV link flows with the deployment of a given number of charging facilities, where the network coverage is defined as the total sum of BEV link flows on only links with charging facility. That is,

$$
\begin{aligned}
\max & \sum_{a} v_{a, e} x_{a} \\
\text { Subject to } & \sum_{a \in A} x_{a}=p .
\end{aligned}
$$

Equation (6) is the budget constraint and can be relaxed as locating the maximum number of $p$ facilities in the network as shown in constraint (7). Consider

$$
0 \leq \sum_{a \in A} x_{a} \leq p
$$

3.2.2. Lower Level Problem. The lower level problem is to obtain the equilibrium BEV flow under SUE routing principle in a congested mixed traffic network considering charging facility locations. The network is assumed to be connected; that is, there is at least one path connecting each O-D pair. We formulate the flow conservation and nonnegativity constraints in the mixed traffic network as follows:

$$
\begin{aligned}
& v_{a}=\sum_{r} \sum_{s} \sum_{k} f_{k g}^{r s} \delta_{a, k}^{r s}+\sum_{r} \sum_{s} \sum_{k} f_{k e}^{r s} \delta_{a, k}^{r s}, \quad \forall a \in A \\
& q_{g}^{r s}=\sum_{k} f_{k g}^{r s}, \quad \forall(r, s) \\
& q_{e}^{r s}=\sum_{k} f_{k e}^{r s}, \quad \forall(r, s) \\
& f_{k g}^{r s} \geq 0, \quad \forall(r, s), k \in K_{g}^{r s} \\
& f_{k e}^{r s} \geq 0, \quad \forall(r, s), k \in K_{e}^{r s} .
\end{aligned}
$$


The link travel cost functions are assumed to be separable between different network links, and they are positive, monotonically increasing, and strictly convex as well. The travel cost for GV drivers includes travel time only, whereas BEV drivers travel cost consists of travel time, charging time, and charging facilities' utility. The perceived path cost is equal to the generalized path travel cost plus a random error term.

$$
\begin{aligned}
& C_{k g}^{r s}=\bar{c}_{k g}^{r s}+\xi_{k g}^{r s}, \quad k \in K_{g}^{r s} \\
& C_{k e}^{r s}=\bar{c}_{k e}^{r s}+\xi_{k e}^{r s}, \quad k \in K_{e}^{r s} .
\end{aligned}
$$

Under SUE, for each O-D pair, GV and BEV flows are distributed on those paths that experience a minimum perceived travel cost and no user can improve its perceived travel cost by unilaterally changing its path. The probability that path $k$ is chosen (by both GV and BEV drivers) can be expressed as

$$
P_{k}^{r s}\left(C_{k}^{r s}\right)=\operatorname{Pr}\left[C_{k}^{r s} \leq C_{r}^{r s}, \forall r \in K^{r s}, r \neq k\right] .
$$

Thus, the SUE path flows are the solution of the following equations:

$$
\begin{aligned}
& f_{k g}^{r s}=q_{g}^{r s} P_{k g}^{r s}\left(C_{k g}^{r s}\right), \quad \forall k \in K_{g}^{r s}, \forall(r, s) \\
& f_{k e}^{r s}=q_{e}^{r s} P_{k e}^{r s}\left(C_{k e}^{r s}\right), \quad \forall k \in K_{e}^{r s}, \quad \forall(r, s) .
\end{aligned}
$$

It has been proved that adding side constraints directly into the general SUE model does not generate the probitbased SUE traffic assignment with side constraints [46]. Jing et al. [47] proposed a solution framework by properly selecting the path set for each O-D pair to ensure the distances of all the used paths are within the BEV range limit with no charging facilities in the network. We extend that SUE model with path-distance constraints to include public charging facilities.

$$
\begin{aligned}
\min _{v_{a}} Z(\mathbf{v}) \\
=-\sum_{r s} q_{g}^{r s} S_{g}^{r s}\left[c^{\mathbf{r s}}(\mathbf{v})\right]-\sum_{r s} q_{e}^{r s} S_{e}^{r s}\left[c^{\mathbf{r s}}(\mathbf{v})\right] \\
+\sum_{a} v_{a} t_{a}\left(v_{a}\right)-\sum_{a} \int_{0}^{v_{a}} t_{a}(\omega) d \omega
\end{aligned}
$$

Subject to $f_{k e}^{r s}\left(D_{e}-l_{k}^{r s, i j}\right) \geq 0$,

$$
\forall(r, s), k \in K_{e}^{r s},(i, j) \in Z_{k}^{r s} .
$$

The objective function (13) of the lower level problem is the classical unconstrained minimization model proposed by Sheffi [48], whose solution is equivalent to SUE conditions satisfying network constraints (8). The novelty of this problem lies in the introduction of subpaths in path selection procedure in constraints (14). It is easy to decide whether a charging action should be taken when arriving at a charging station to make sure BEVs can reach the next charging station or destination; namely, the subpath distance $l_{k}^{r s, i j},(i, j) \in$ $Z_{k}^{r s}$, of path $k \in K_{e}^{r s}$ is less than $D_{e}$. Supposing that there are $Z_{L}$ charging stations deployed along a path for BEVs, only less than $2^{Z_{L}}$ charging decision should be made and
$c_{Z_{L}+2}^{2}$ subpaths exist when going through this path. Therefore, by comparing the driving distance limit $D_{e}$ with subpath distance $l_{k}^{r s, i j}$, the set of feasible subpaths generated from finite paths between each O-D pair can be predetermined. The generation of feasible subpaths is illustrated in Figure 1 which is similar to the way of predetermining battery-swapping action based feasible paths in Xu et al. [49]. First we prove the equivalence between the solution of the proposed model (see (13)) and SUE solution. The Lagrangian function can be written as

$$
\begin{aligned}
L(\mathbf{v}, \boldsymbol{\mu})= & -\sum_{r} \sum_{s} q_{g}^{r s} S_{g}^{r s}\left[c^{\mathbf{r s}}(\mathbf{v})\right]-\sum_{r} \sum_{s} q_{e}^{r s} S_{e}^{r s}\left[c^{\mathbf{r s}}(\mathbf{v})\right] \\
& +\sum_{a} x_{a} t_{a}\left(x_{a}\right)-\sum_{a} \int_{0}^{x_{a}} t_{a}(\omega) d \omega \\
& -\sum_{r} \sum_{s} \sum_{k} \mu_{k e}^{r s, i j} \cdot f_{k e}^{r s} \cdot\left(D_{e}-l_{k}^{r s, i j}\right),
\end{aligned}
$$

where $\mu_{k e}^{r s, i j}$ is the Lagrangian multiplier corresponding to path/subpath-distance constraint (14). $\mu_{k e}^{r s, i j} f_{k e}^{r s} \cdot\left(D_{e}-l_{k}^{r s, i j}\right)$ can be perceived as the path out-of-range cost incurred when the path/subpath distance exceeds the driving distance limit of the BEV and it should fulfill the following conditions:

$$
\begin{aligned}
& \mu_{k e}^{r s, i j}=0, \quad \text { if } l_{k}^{r s, i j} \leq D_{e} \\
& \mu_{k e}^{r s, i j} \geq 0, \quad \text { if } l_{k}^{r s, i j}>D_{e} .
\end{aligned}
$$

If the flow of BEV drivers going through this path is positive, the path/subpath distance is smaller than or equal to the driving distance limit; otherwise, the trip flow is zero. $\mu_{k e}^{r s, i j}$ is the unit path/subpath out-of-range cost.

The first-order derivative of (13) must satisfy the SUE conditions. Let

$$
\nabla L(\mathbf{v}, \boldsymbol{\mu})=0 .
$$

The gradient with respect to link flow vector is

$$
\begin{aligned}
& \frac{\partial L(\mathbf{v}, \boldsymbol{\mu})}{\partial v_{b}} \\
& =\left(-\sum_{r} \sum_{s} \sum_{k \in K_{g}^{r s}} q_{g}^{r s} P_{k g}^{r s} \delta_{b, k}^{r s}-\sum_{r} \sum_{s} \sum_{k \in K_{e}^{r s}} q_{e}^{r s} P_{k e}^{r s} \delta_{b, k}^{r s}+v_{b}\right) \frac{d t_{b}}{d v_{b}} \\
& \quad-\sum_{r} \sum_{s} \sum_{k \in K_{e}^{r s}} \mu_{k e}^{r s, i j} \cdot\left(D_{e}-l_{k}^{r s, i j}\right) \delta_{b, k}^{r s} .
\end{aligned}
$$

Note that the extra path/subpath-distance constraints could be infeasible if the distance of any selected subpath exceeds the BEVs' driving distance limit. If all the selected paths and their subpaths are within driving distance limit, the subpath out-of-range cost $\mu_{k e}^{r s, i j} \cdot\left(D_{e}-l_{k}^{r s, i j}\right)$ should be equal to zero. The derivative of the SUE objective function becomes

$$
\begin{aligned}
& \frac{\partial L(\mathbf{v}, \boldsymbol{\mu})}{\partial v_{b}} \\
& =\left(-\sum_{r} \sum_{s} \sum_{k \in K_{g}^{r s}} q_{g}^{r s} P_{k g}^{r s} \delta_{b, k}^{r s}-\sum_{r} \sum_{s} \sum_{k \in K_{e}^{r s}} q_{e}^{r s} P_{k e}^{r s} \delta_{b, k}^{r s}+v_{b}\right) \frac{d t_{b}}{d v_{b}} .
\end{aligned}
$$


The gradient equals zero if and only if

$$
v_{b}=\sum_{r} \sum_{s} \sum_{k \in K_{e}^{r s}} q_{e}^{r s} P_{k e}^{r s} \delta_{b, k}^{r s}+\sum_{r} \sum_{s} \sum_{k \in K_{g}^{r s}} q_{g}^{r s} P_{k g}^{r s} \delta_{b, k}^{r s}
$$

$\forall b \in A$.

Equation (20) expresses the SUE link flows consisting of $\mathrm{BEV}$ and GV flows and the feasible solution can be ensured by properly selecting paths. Then we can prove the Hessian matrix of the SUE objective function is positive definite, because the second derivative of $\sum_{r} \sum_{s} \sum_{k \in K_{e}^{r s}} \mu_{k e}^{r s, i j} \cdot f_{k e}^{r s} \cdot\left(D_{e}-\right.$ $l_{k}^{r s, i j}$ ) with respect to path flow equals zero. This proves that the resulting SUE link flow pattern is unique.

\section{Solution Method}

The bilevel programming problem is NP-hard. Thus, we propose an equilibrium-based heuristic to iteratively solve the lower level SUE problem and the upper level problem. The interaction between the upper and lower levels, shown in Figure 2, captures the effect of charging facility location on the routing behavior of BEV drivers, which further determines the BEV and GV flow patterns. Initially, we assume no charging facilities in the network. The lower level problem is a stochastic traffic assignment of mixed GV and BEV flows under path-distance constraints. After the first run of the lower level problem, we can obtain the initial BEV link flow pattern. The upper level problem then finds the best $p$ charging facility locations to maximize the total covered $\mathrm{BEV}$ flow. The obtained charging facility locations will be compared with the previous location solutions. If there is no change in charging facility location, the procedure ends with the current solution; otherwise, the lower level SUE assignment is repeated with updated charging facility locations.

The detailed procedure is as follows. Note that a Multinomial Logit choice model is used in the lower level SUE TAP.

Step 1. Set upper level iteration counter $z=1$. Input initial charging facility location, namely, no charging facility in the network. Relax BEVs' distance constraints and perform conventional SUE assignment to identify the corresponding SUE link flow pattern.

Step 2. Increase the upper level iteration counter by 1 . Sort all the links in ascending order of their BEV flows and find the top $p$ of them. Locate the charging facilities (uncapacitated) in the middle of the $p$ links.

Step 3. Perform SUE assignment with charging facilities in the network from Step 2. The detailed steps are listed below.

Step 3.1 (subpath feasibility check). Set $x_{a}(0)=0$ and $t_{a}=$ $t_{a}\left[x_{a}(0)\right]$. For each O-D pair, find $K$ shortest paths for both GVs and BEVs in terms of free-flow travel time and record them as initial path set. For each path of BEVs, identify the path distance, the number of charging facilities on this path, the location of charging facilities, and pure subpath distances. If any pure subpath distance is greater than the BEVs' driving distance limit, set its corresponding path travel time to infinity and this path becomes infeasible. If all the $K$ paths are infeasible, record this O-D pair to Set $A$. If Set $A$ is empty which means there exists at least one feasible path between each O-D pair, go to the next step; otherwise, stop.

Step 3.2 (initialization). Calculate the generalized BEV path travel cost $\bar{c}_{k e}^{r s}$ and the probability of choosing each path to get the auxiliary link flow pattern. Perform stochastic network loading to assign the entire demand of each class of vehicles between each O-D pair to the corresponding $K$ shortest paths. This yields $\mathbf{v}_{a, g}(1)$ and $\mathbf{v}_{a, e}(1)$. Set iteration counter $n=1$.

Step 3.3 (update). Calculate a new link cost in terms of $t_{a}=$ $t_{a}\left[\mathbf{v}_{\mathbf{a}}(1)\right], \forall a$.

Step 3.4 (direction finding). Follow the same procedure described in Step 3.1 to find $K$ shortest path for each class of vehicles based on the current set of link travel times, $\left\{t_{a}^{n}\right\}$. If all the pure subpaths of the generated $K$ paths between an O-D pair exceed the range limit, use initial path set in Step 3.1 and perform stochastic network loading. This yields an auxiliary link flow pattern $\left\{y_{a, g}\right\},\left\{y_{a, e}\right\}$.

Step 3.5 (step size). A predetermined step size sequence $\left\{\alpha_{n}\right\}$ is used: $\alpha_{n}=1 / n, n=1,2, \ldots, \infty$.

Step 3.6 (move). Find the new flow pattern by setting

$$
\begin{aligned}
& \mathbf{v}_{a}^{n+1}=\mathbf{v}_{a}^{n}+\left(\frac{1}{n}\right)\left(\mathbf{y}_{a}^{n}-\mathbf{v}_{a}^{n}\right) \\
& \mathbf{v}_{a, g}^{n+1}=\mathbf{v}_{a, g}^{n}+\left(\frac{1}{n}\right)\left(\mathbf{y}_{a, g}^{n}-\mathbf{v}_{a, g}^{n}\right) \\
& \mathbf{v}_{a, e}^{n+1}=\mathbf{v}_{a, e}^{n}+\left(\frac{1}{n}\right)\left(\mathbf{y}_{a, e}^{n}-\mathbf{v}_{a, e}^{n}\right) .
\end{aligned}
$$

Step 3.7 (convergence test). Let

$$
\bar{v}_{a}^{n}=\frac{1}{m}\left(v_{a}^{n}+v_{a}^{n-1}+\cdots+v_{a}^{n-m+1}\right) .
$$

If the convergence criterion

$$
\frac{\sqrt{\sum_{a}\left(\bar{v}_{a}^{n+1}-\bar{v}_{a}^{n}\right)^{2}}}{\sum_{a} \bar{v}_{a}^{n}} \leq \kappa
$$

is met, stop and $\left\{\mathbf{v}_{a}^{n+1}\right\},\left\{\mathbf{v}_{a, e}^{n+1}\right\}$ are the sets of equilibrium link flows and BEV link flows, respectively; otherwise, set $n=n+1$ and go to Step 3.3.

Step 4. Repeat Step 2 and update the current charging facility location. Compare the current location with previous location status at Step 2. If the locations do not change, stop and record the current charging facility location; otherwise, go to Step 3. 

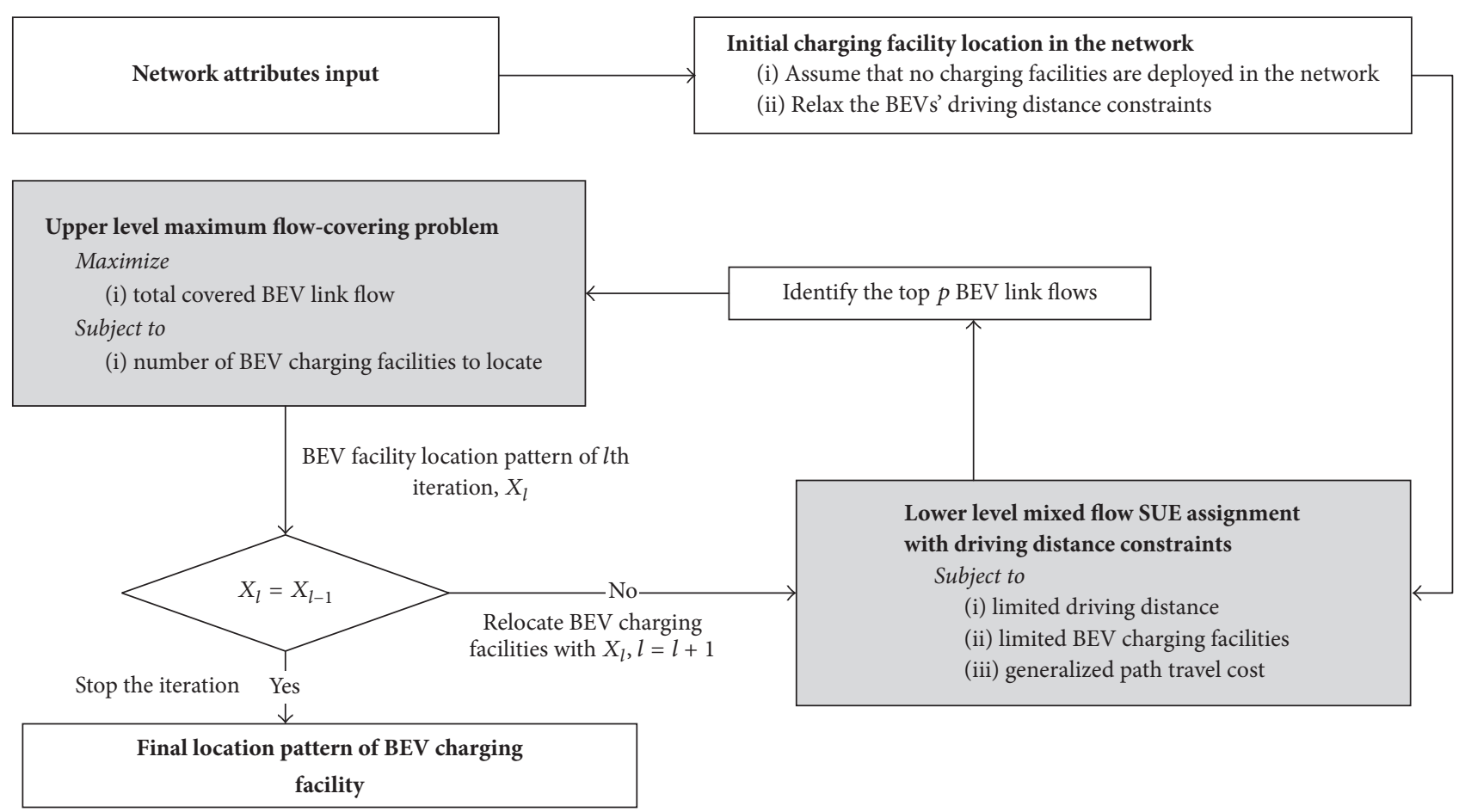

FIGURE 2: Framework of the bilevel proposed method for the equilibrium-optimization-based BEV charging facility location problem.

TABLE 1: O-D demand of Nguyen-Dupuis network.

\begin{tabular}{lcc}
\hline O-D & BEV & GV \\
\hline$(1,2)$ & 200 & 200 \\
$(1,3)$ & 400 & 400 \\
$(4,2)$ & 300 & 300 \\
$(4,3)$ & 100 & 100 \\
\hline
\end{tabular}

\section{Numerical Analysis}

This section presents the numerical results of the model and solution algorithm applied to two network case studies. The analysis aims at assessing the impacts of charging facility utility, charging speed, and driving distance limit on the optimal placement of charging facility locations.

The first numerical example is the Nguyen-Dupuis network; see, for example, [49]. The network consists of 13 nodes, 19 links, and 4 O-D pairs: $(1,2),(1,3),(4,2)$, and $(4,3)$, as shown in Figure 3. The network supply and O-D demands information are from Nguyen and Dupuis [50]. The O-D demand is assumed to be the same for both GVs and BEVs; that is, BEV market penetration rate is $50 \%$ (given in Table 1 ) to facilitate the equilibrium flow comparison between $\mathrm{BEV}$ and GV. The free-flow travel time is used as a proxy for the link length for each link. Due to the small size of the NguyenDupuis network, the enumerated path sets information is obtained from Jiang and Xie [43] in Table 2.

We use this case study to evaluate the performance of the proposed algorithm for solving the bilevel model where lower level problem is logit-based SUE assignment with driving

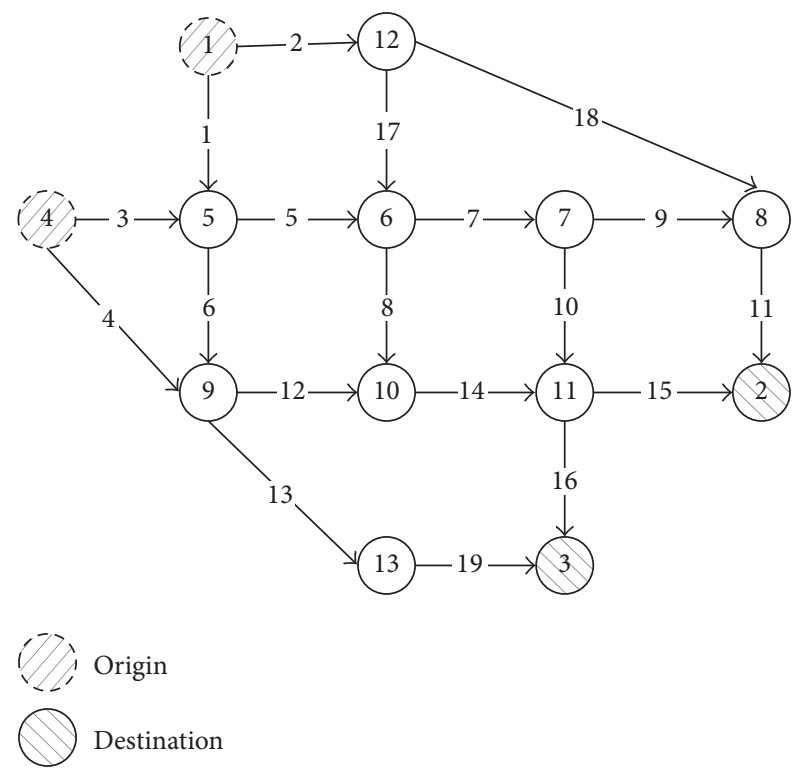

FIGURE 3: The Nguyen-Dupuis network with 2 origins, 2 destinations, 13 nodes, 19 links, and 25 paths between the 4 O-D pairs.

distance constraints. The following parameter values are considered. We do not claim the suitability of the defined parameters for accurate quantification of network performance. To avoid the dominant role of $t_{u}^{0}$ in the path cost, a relatively small proportion of charging facility is deployed in this 19link network: $p=3$. The BEV driving distance limit is set to 20 , the scale parameters of the logit model for route choice of 
TABle 2: Path compositions and lengths in the Nguyen-Dupuis network example.

\begin{tabular}{|c|c|c|c|}
\hline $\mathrm{O}-\mathrm{D}$ & Path number & Path composition & Length \\
\hline \multirow{8}{*}{$(1,2)$} & 1 & $1-5-6-7-8-2$ & 29 \\
\hline & 2 & $1-5-6-7-11-2$ & 33 \\
\hline & 3 & $1-5-6-10-11-2$ & 38 \\
\hline & 4 & $1-5-9-10-11-2$ & 41 \\
\hline & 5 & $1-12-6-7-8-2$ & 35 \\
\hline & 6 & $1-12-6-7-11-2$ & 39 \\
\hline & 7 & $1-12-6-10-11-2$ & 44 \\
\hline & 8 & $1-12-8-2$ & 32 \\
\hline \multirow{6}{*}{$(1,3)$} & 9 & $1-5-6-7-11-3$ & 32 \\
\hline & 10 & $1-5-6-10-11-3$ & 37 \\
\hline & 11 & $1-5-9-10-11-3$ & 40 \\
\hline & 12 & $1-5-9-13-3$ & 36 \\
\hline & 13 & $1-12-6-7-11-3$ & 38 \\
\hline & 14 & $1-12-6-10-11-3$ & 43 \\
\hline \multirow{5}{*}{$(4,2)$} & 15 & $4-5-6-7-8-2$ & 31 \\
\hline & 16 & $4-5-6-7-11-2$ & 35 \\
\hline & 17 & $4-5-6-10-11-2$ & 40 \\
\hline & 18 & $4-5-9-10-11-2$ & 43 \\
\hline & 19 & $4-9-10-11-2$ & 37 \\
\hline \multirow{6}{*}{$(4,3)$} & 20 & $4-5-6-7-11-3$ & 34 \\
\hline & 21 & $4-5-6-10-11-3$ & 39 \\
\hline & 22 & $4-5-9-10-11-3$ & 42 \\
\hline & 23 & $4-5-9-13-3$ & 38 \\
\hline & 24 & $4-9-10-11-3$ & 36 \\
\hline & 25 & $4-9-13-3$ & 32 \\
\hline
\end{tabular}

GV and BEV are $\gamma_{g}=\gamma_{e}=0.1$, the charging speed is $\varepsilon=1$, the utility of a charging facility on path is $t_{u}^{0}=-2$, and $K$ in the $K$ shortest paths is set to be 5 . In addition, the link capacity and free-flow travel time (link length) are given in Table 3 with the equilibrium BEV link flow at each upper level iteration.

The relationship between charging facility location pattern in the upper level and BEV link flows in the lower level is first examined. Table 3 lists the charging facility locations and the corresponding BEV link flows in each iteration. At the first iteration, we assume no charging facility is available in the network and relax the driving distance constraints. The results clearly show the overall BEV link flow pattern in the first iteration is quite different from those in the others, especially after the first iteration when charging facilities are located in the network. In the first iteration, every enumerated path is feasible for BEV drivers since the driving distance constraint is relaxed. As for the other iterations, some paths become infeasible due to the lack of charging facilities. For example, only path 18 between O-D pair $(4,2)$ is feasible in the last iteration because two charging stations are deployed on links 6 and 14 so that each pure subpath distance is smaller than the range limit.

The total covered flows by locating 3 charging facilities in this example are " $0,1054.3,1048.5$, and 1048.5" during the four iterations. The amount of total covered BEV flows in the third iteration may decrease comparing to the second iteration because the BEV flow covered in the second iteration is actually generated by using the charging facility locations in the first iteration. Therefore, when new locations are generated, the BEV link flow changes accordingly until the last two iterations that produce the same facility locations. The potential drawback of this modified definition of maximum covering flow is that if a route contains multiple links with charging stations (e.g., paths 4 and 11), a trip by a driver is counted multiple times even though BEV drivers may not charge or only charge once during the trip. As a result, this method could locate charging facilities on several adjacent links of some high-volume freeways, while in practice fast charging facilities are usually deployed with long intradistances along the freeways.

A sensitivity analysis is conducted with respect to the charging facility utility, charging speed, and BEV driving distance limit. The results are illustrated in Figure 4, where only one parameter is changed in each scenario. In scenario (a), we set the charging speed as $\varepsilon=0.1$ which can be regarded as relatively fast charging and we conduct tests on different level of charging facility utility. The utility value can be perceived as the risk-taking level of BEV drivers. A smaller utility value indicates that BEV drivers are willing to take more risks. As the equivalent travel time reduction value (i.e., utility) goes up, the total covered BEVs flows increases, because BEVs drivers are more likely to choose feasible lengthy paths with fast charging facilities instead of paths with less travel time. If we consider multiple classes of $\mathrm{BEV}$ drivers with different driving distance limits, the BEVs with shorter driving distance and risk-neutral attitude would probably have a larger value of charging facilities utility, because charging facilities help to ease their range anxiety, while, for those with larger batteries, they would behave more like GV users. In general, large travel time reduction value should apply to fast charging method, small battery capacities, and risk-taking BEV drivers.

We then examine the impacts of charging speed, that is, $\varepsilon$, in scenario (b), where a smaller value represents a faster charging speed, with charging time estimated as $t_{c, k}^{r s}=\varepsilon$. $\left(l_{k}^{r s, r s}-D_{e}\right)$. This parameter translates to different charging methods (i.e., slow charging, fast charging, or batteryswapping technology) that lead to different charging facility location patterns. Given a charging facility location pattern (e.g., $\{1,5,7\})$, charging speed affects the total travel cost on a feasible path. As a result, the probability of choosing each path changes if there exist at least two feasible paths between each O-D pair. With $\varepsilon=0.01$, the charging facilities are deployed on link $\{1,5,7\}$ and the feasible paths are paths 9 and 13 between O-D pair $(1,3)$, whereas, with $\varepsilon=10$, the charging facilities are located on $\{6,12,14\}$. Only path 11 is feasible between O-D pair $(1,3)$, and all the BEV drivers will be assigned to this path if no other paths are feasible. In this case, charging speed does not affect the path choice probability. Fast charging attracts more BEV flows compared to slow charging when at least one another path with no charging need is available to BEV users, because the charging speed would have the influence on the total travel cost and path choice probability only if $\mathrm{BEV}$ drivers take charging 
TABLE 3: The charging facility locations and BEV flows over iterations.

\begin{tabular}{|c|c|c|c|c|c|c|c|c|c|c|c|}
\hline \multirow{2}{*}{\multicolumn{2}{|c|}{ Link number }} & \multirow{3}{*}{ Link length } & \multirow{3}{*}{ Link capacity } & \multicolumn{8}{|c|}{ Upper level iteration } \\
\hline & & & & \multicolumn{2}{|c|}{1} & \multicolumn{2}{|c|}{2} & \multicolumn{2}{|c|}{3} & \multicolumn{2}{|c|}{4} \\
\hline & & & & Location & BEV flow & Location & BEV flow & Location & BEV flow & Location & BEV flow \\
\hline$(1,5)$ & 1 & 7 & 500 & I & 349.8 & $\sqrt{ }$ & 316.2 & $\sqrt{ }$ & 335.5 & $\sqrt{ }$ & 335.5 \\
\hline$(1,12)$ & 2 & 9 & 500 & l & 250.2 & l & 283.8 & l & 264.5 & l & 264.5 \\
\hline$(4,5)$ & 3 & 9 & 500 & l & 257.1 & l & 188.0 & I & 194.5 & I & 194.5 \\
\hline$(4,9)$ & 4 & 12 & 400 & l & 142.9 & I & 212.0 & I & 205.5 & I & 205.5 \\
\hline$(5,6)$ & 5 & 3 & 500 & l & 395.4 & $\sqrt{ }$ & 202.5 & l & 228.1 & l & 228.1 \\
\hline$(5,9)$ & 6 & 9 & 500 & I & 211.5 & l & 301.6 & $\sqrt{ }$ & 301.8 & $\sqrt{ }$ & 301.8 \\
\hline$(6,7)$ & 7 & 5 & 500 & l & 404.4 & $\sqrt{ }$ & 172.5 & I & 196.5 & I & 196.5 \\
\hline$(6,10)$ & 8 & 13 & 500 & I & 159.0 & I & 175.7 & I & 158.1 & I & 158.1 \\
\hline$(7,8)$ & 9 & 5 & 500 & I & 161.4 & I & 75.4 & I & 87.8 & I & 87.8 \\
\hline$(7,11)$ & 10 & 9 & 500 & I & 243.0 & I & 97.2 & I & 108.7 & I & 108.7 \\
\hline$(8,2)$ & 11 & 9 & 500 & I & 243.5 & I & 213.5 & I & 225.9 & I & 225.9 \\
\hline$(9,10)$ & 12 & 10 & 500 & I & 164.7 & I & 260.8 & I & 253.0 & I & 253.0 \\
\hline$(9,13)$ & 13 & 9 & 400 & I & 189.7 & I & 252.9 & I & 254.3 & I & 254.3 \\
\hline$(10,11)$ & 14 & 6 & 500 & I & 323.8 & I & 436.5 & $\sqrt{ }$ & 411.2 & $\sqrt{ }$ & 411.2 \\
\hline$(11,2)$ & 15 & 9 & 500 & I & 256.5 & I & 286.5 & I & 274.1 & I & 274.1 \\
\hline$(11,3)$ & 16 & 8 & 500 & I & 310.3 & I & 247.1 & I & 245.7 & I & 245.7 \\
\hline$(12,6)$ & 17 & 7 & 500 & I & 168.1 & I & 145.7 & I & 126.5 & I & 126.5 \\
\hline$(12,8)$ & 18 & 14 & 400 & I & 82.1 & I & 138.1 & I & 138.0 & I & 138.0 \\
\hline$(13,3)$ & 19 & 11 & 500 & I & 189.7 & I & 252.9 & I & 254.3 & I & 254.3 \\
\hline
\end{tabular}

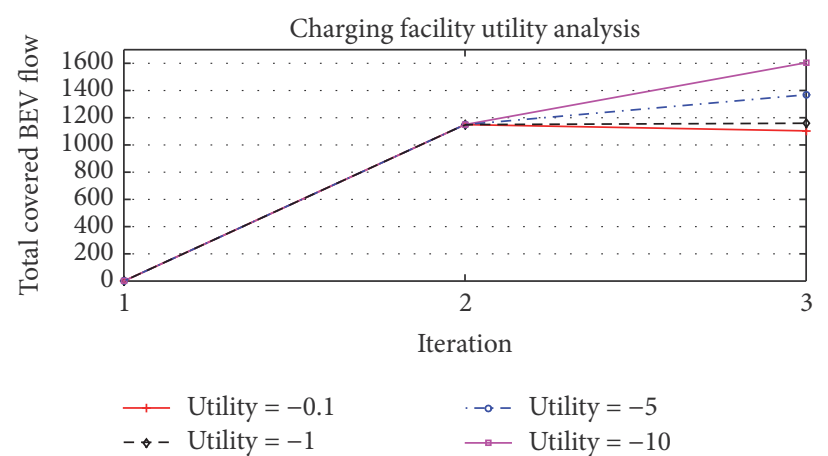

(a) Utility

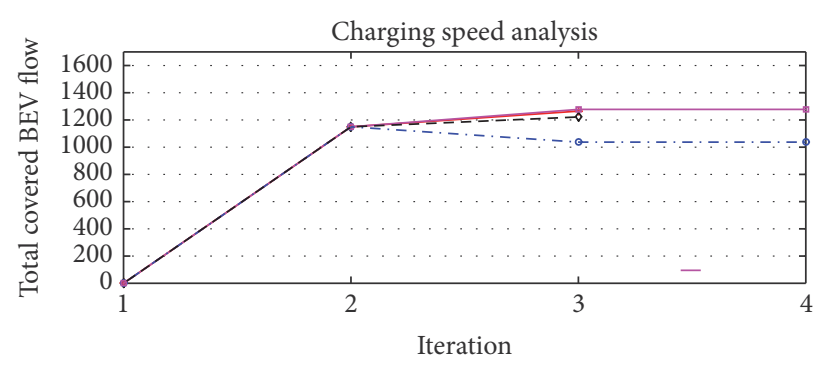

$\leftarrow$ Charging speed $=0.01 \quad-\propto-$ Charging speed $=1$

$-\rightarrow$ Charging speed $=0.1 \rightarrow$ Charging speed $=10$

(b) Charging speed

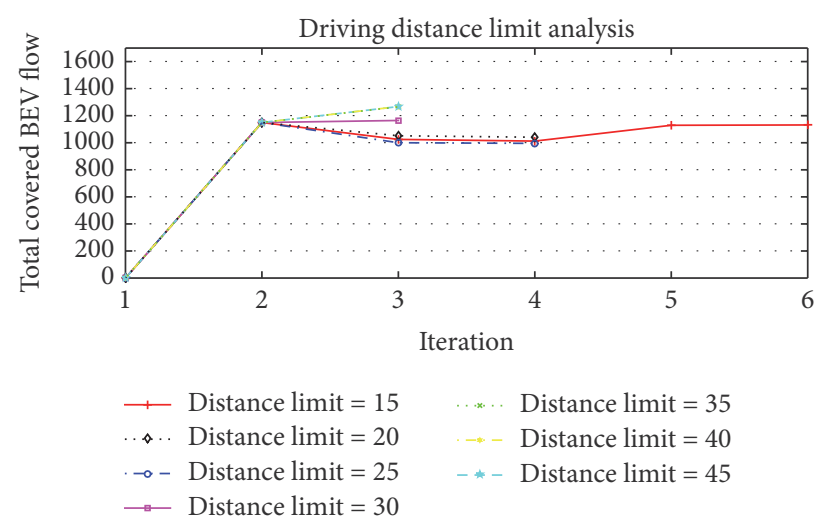

(c) Driving distance limit

FIGURE 4: Sensitivity analysis for various input parameters (a) charging facility utility; (b) charging speed; and (c) driving distance limit on the total covered BEV flows. 
action with these facilities. The generalized travel cost on paths with charging actions would be too high when charging speed is extremely slow (e.g., $\varepsilon=10$ ) and charging time takes over path travel time. BEVs would probably choose saturated paths with high travel time. However, as can be seen from the results, the total covered $\mathrm{BEV}$ flow is not strictly increasing with the increase of charging speed and it is also influenced by the feasible path set between O-D pairs.

In scenario (c), the lower bound of distance limit is set to 15 to make sure there exists at least one feasible path between each O-D pair. In addition, given that all paths are enumerated in Table 2, the distance limit 45 is the path length upper bound in the network without imposing the distance limit. The charging facility locations for distance limits 15 , 20 , and 25 are $\{5,7,14\},\{1,6,14\}$, and $\{1,5,14\}$, respectively, and the total covered BEV flows are 1131.6, 1040.9, and 995.5. Additionally, the charging facilities are all located on $\{1,5,7\}$ for distance limits $30,35,40$, and 45 , covering, respectively, $1164.7,1265.4,1267.5$, and 1267.6 BEV flows. It is observed that as the distance limit increases, the total covered BEV flow decreases at first, while after the distance limit reaches a certain value, the total covered BEV flows increase till it reaches a stable value. The driving distance limit affects the number of feasible subpaths and charging time. As the driving distance limit increases, more paths are eligible to carry flows and a larger $K$ value should be used to generate more feasible paths during the assignment process. However, as indicated in [38], the change in the number of feasible paths does not always increase with the distance limit, since each subpath of the generated $K$ shortest paths would be feasible when the distance limit is large enough.

From the three sensitivity analysis scenarios, it is observed that the proposed model can satisfy the stopping criteria after 3 or 4 iterations for this small network. Although there is no significant difference in the total covered BEV flows, the charging facility locations vary for each scenario. It is noteworthy that the deployment of charging facilities changes BEV path flow patterns while the aggregated covered BEV link flows do not change significantly. Therefore, the strategy of locating charging facilities is still focusing on those $\mathrm{BEV}$ saturated links to increase the exposure of charging facilities to BEV flows. Taking realistic situation into consideration, when budget is limited, the number of charging facilities can be flexible by adjusting its size and configuration. It would be better to scatter more small size charging facilities than large ones to increase the exposure to BEV drivers. The charging speed affects the BEVs perceived travel cost only when they need charging. Thus fast charging station or chargers should be deployed along freeways or highways to reduce the charging time of long-distance trips while slow chargers can be deployed along urban roads to eliminate range anxiety and to increase exposure. Under some circumstances, charging station equipped both slow and fast chargers may enable more flexible charging operation. We also found that the BEVs are restricted to some relatively short paths especially when distance limit is low; however, the equilibrium mechanism will assign more GVs to relatively long paths since the GV drivers still try to minimize their perceived travel time.
The second numerical experiment is done on the Sioux Falls network shown in Figure 5, which has been chosen as a benchmark network in numerous traffic assignment studies. We adopt a variation of this network presented in Suwansirikul et al. [51]. The exact network attributes and travel demands are also used in our study. For simplicity, the free-flow travel time is used as proxy for link length and BEV penetration rate is assumed to be 50\%. Sioux Falls network consists of 24 nodes, 76 links, and $576 \mathrm{O}-\mathrm{D}$ pairs. The number of charging facilities is $p=8$. This example is to evaluate the computational performance of the proposed solution algorithm. For computational experiments, the number of iterations (ITR) and the total computational cost (TCC) were compared under different parameter settings.

Table 4 lists the computational cost under different parameter settings. Assuming the logit scaling parameter be 0.1 , it can be seen from Scenario 1 that the computational cost generally increases as the driving distance limit increases. The underlying reason might be that many paths become feasible in the $K$ paths generated, thus requiring the related path/subpath choice probability calculation and assignment. From the first two scenarios, clearly $K$ value has an impact on the computational cost, because bigger $K$ value would increase the computational time in the $K$ shortest path algorithm as well as the stochastic network loading procedure in the lower level problem. Comparing Scenario 2 with Scenarios 3 and 4, respectively, the results demonstrate that charging speed and charging facilities' utility affect computational time marginally. Finally, we can observe that $K$ value has the most impact on increasing computational time and the number of iterations needed for the upper level problem.

\section{Conclusions and Future Work}

This paper formulates, solves, and evaluates the problem of potential location of public charging facilities for BEV in a network with mixed GVs and BEVs. The path travel cost of BEVs is modeled by considering path travel time, charging time, driving distance limit, and charging facilities' utility, where driving distance limit restricts the path choice. A bilevel model has been proposed to address the issue of coexisting equilibrium GV-BEV flows. A mix-integer nonlinear program is constructed based on MSA to maximize the total BEV flow coverage on high-BEV-traffic paths. The key part of this formulation is the lower level path-distance constrained stochastic traffic assignment. The solution equivalency is proved to satisfy SUE condition as well as the uniqueness of link flow pattern. Moreover, a modified MSA method with $K$ shortest path algorithm and generalized BEV path travel cost are applied to solve the charging facility location problem. In the numerical analysis, we also demonstrated how the driving distance limits, charging speed, and utility of charging facilities affect the equilibrium network flow and charging facility location.

We expect that the strategy of locating charging facilities and the modeling technique presented in this work would potentially trigger the interest of incorporating other types of BEV-specific constraints in the lower level problem, such 
TABLE 4: Computational cost with different parameter settings for MNL.

\begin{tabular}{lccccccc}
\hline & \multicolumn{3}{c}{ Scenario $1: K=3, \varepsilon=1, t_{u}^{0}=0.001$} & \multicolumn{3}{c}{ Scenario $2: K=5, \varepsilon=1, t_{u}^{0}=0.01$} \\
\hline$D_{e}$ & 0.25 & 0.4 & 0.6 & 0.8 & 0.25 & 0.4 & 0.6 \\
ITR & 5 & 3 & 3 & 3 & 4 & 4 & 3 \\
TCC(s) & 117.5 & 168.74 & 166.56 & 173.15 & 197.33 & 474.45 & 328.17 \\
\hline \multicolumn{3}{r}{} & Scenario $3: K=5, \varepsilon=0.1, t_{u}^{0}=0.01$ & & Scenario $4: K=5, \varepsilon=1, t_{u}^{0}=0.001$ & 329.46 \\
\hline$D_{e}$ & 0.25 & 0.4 & 0.6 & 0.8 & 0.25 & 0.4 & 0.6 \\
ITR & 4 & 4 & 3 & 3 & 4 & 4 & 3 \\
TCC(s) & 199.18 & 477.28 & 328.06 & 326.61 & 198.15 & 475.62 & 327.09 \\
\hline
\end{tabular}

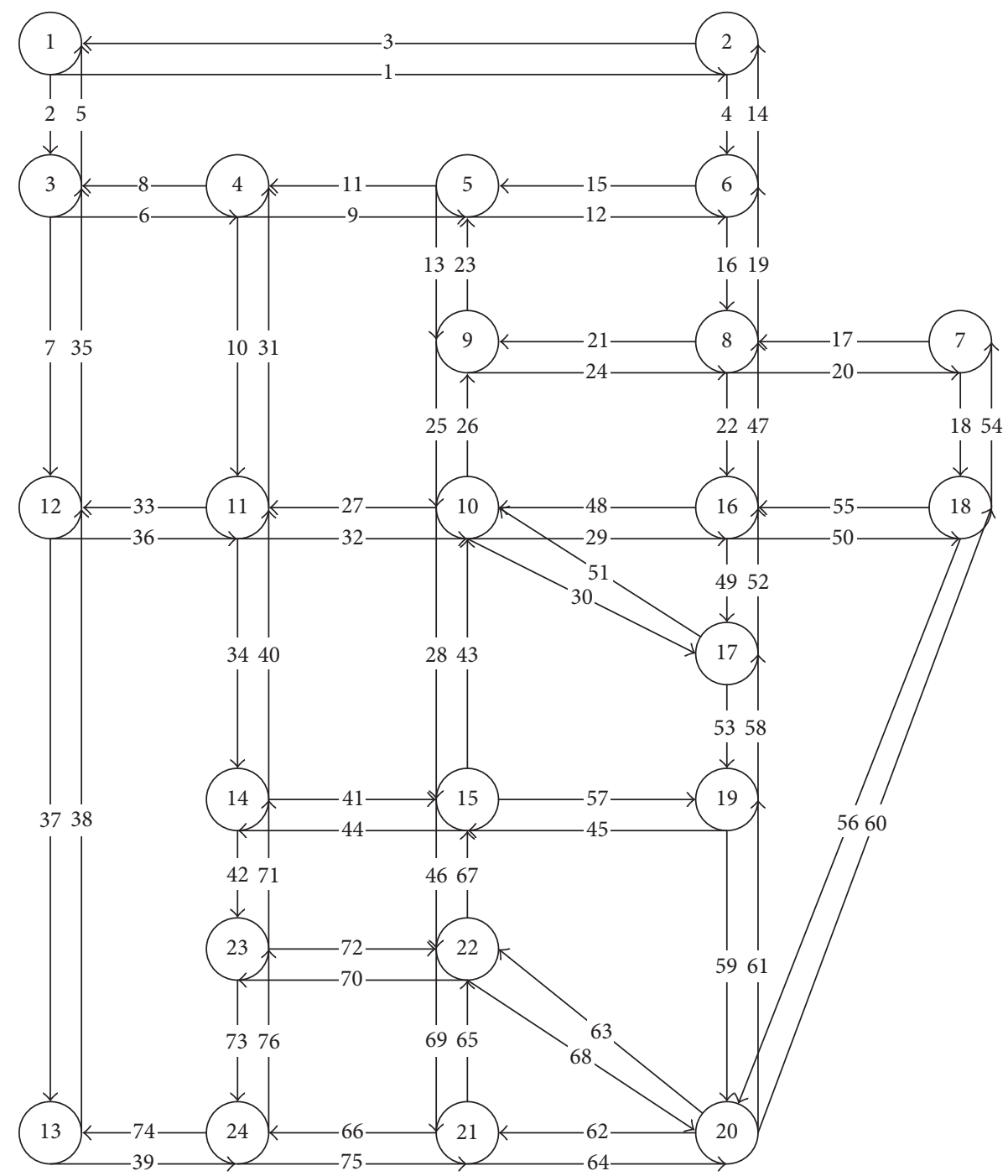

FIgURE 5: Sioux Falls network with 24 nodes and 76 links.

as flow-dependent battery capacity constraints and timedependent battery-charging price. As for the upper level problem, some other approaches, such as FILM and FRLM, locating charging facilities to maximize passing BEV flows without double counting, can be explored to better serve the BEV travel demand. The model uses a number of assumptions to simplify the problem and make it tractable, which will be relaxed in the future work to deal with more complicating and realistic issues.

\section{Notations}

$K_{g}^{r s}, K_{e}^{r s}$ : Set of paths connecting O-D pair $(r, s)$ of $\mathrm{GV}$ and BEV, respectively 


\begin{tabular}{|c|c|}
\hline$r s$ & $\begin{array}{l}\text { Set of pseudonodes of charging stations on } \\
\text { path } k \in K_{e}^{r s} \text { connecting O-D pair }(r, s)\end{array}$ \\
\hline $\begin{array}{ll}r s, i j \\
k\end{array}$ & $\begin{array}{l}\text { Length of subpath } k^{i j} \text { in path } \\
k,(i, j) \in Z_{k}^{r s}\end{array}$ \\
\hline$l_{a}:$ & Length of link $a, a \in A$ \\
\hline$v_{a}:$ & $\begin{array}{l}\text { Traffic flow on link } a \in A \text {, which is the } \\
\text { summation of GV link flow } v_{a, g} \text { and BEV } \\
\text { link flow } v_{a, e} \text { that is, } v_{a}=v_{a, g}+v_{a, e}\end{array}$ \\
\hline v: & $\begin{array}{l}\text { A column vector of all the link flows; } \\
\mathbf{v}=\left(v_{a}\right)^{T}, a \in A\end{array}$ \\
\hline$x_{a}:$ & $\begin{array}{l}\text { Binary variable, equaling } 1 \text { if there is a } \\
\text { charging facility at location } z \in Z \text { on link } \\
a 0 \text { otherwise }\end{array}$ \\
\hline $\mathbf{x}$ : & $\begin{array}{l}\text { A column vector of all the location } \\
\text { variables; } \mathbf{x}=\left(x_{a}\right)^{T}, a \in A\end{array}$ \\
\hline & Link travel time on link $a$ \\
\hline & $\begin{array}{l}\text { Link path incidence: } \delta_{a, k}^{r s}=1 \text { if path } \\
k \in K_{g}^{r s}, K_{e}^{r s} \text { between O-D pair }(r, s) \\
\text { traverses link } a \text { and } 0 \text { otherwise }\end{array}$ \\
\hline$l_{k}^{r s}:$ & $\begin{array}{l}l_{k}^{r s}=\sum_{a} l_{a} \delta_{a, k}^{r s} \text {, length of path } k \text { between } \\
\text { O-D pair }(r, s)\end{array}$ \\
\hline & Driving distance limit of $\mathrm{BEV}$ \\
\hline$f_{k g}^{r s}, f_{k e}^{r s}:$ & $\begin{array}{l}\text { Traffic flow of GV and BEV on path } \\
k \in K_{g}^{r s}, K_{e}^{r s}\end{array}$ \\
\hline$c_{k}^{r s}(\mathbf{f}):$ & $\begin{array}{l}\text { Path } k \text { travel time between O-D pair } \\
(r, s), k \in K_{e}^{r s}, K_{g}^{r s} ; c_{k}^{r s}(\mathbf{f})=\sum_{a} t_{a}\left(v_{a}\right) \delta_{a, k}^{r s}\end{array}$ \\
\hline & Total travel time reduction on path $k \in K_{e}^{r s}$ \\
\hline$t_{u}^{0}:$ & $\begin{array}{l}\text { The utility of one charging facility on the } \\
\text { path, equivalent to a constant nonpositive } \\
\text { travel time reduction value }\end{array}$ \\
\hline $\bar{c}_{k g}^{r s}, \bar{c}_{k e}^{r s}$ & $\begin{array}{l}\text { Generalized travel cost of } \mathrm{GV} \text { or } \mathrm{BEV} \text { on a } \\
\text { given path } k \in K_{g}^{r s}, K_{e}^{r s}\end{array}$ \\
\hline 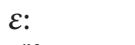 & Battery-charging speed, $\mathrm{min} / \mathrm{km}$ \\
\hline$t_{c, k}^{r s}:$ & $\begin{array}{l}\text { Charging time needed on a given path } \\
k \in K_{e}^{r s} \text { between O-D pair }(r, s)\end{array}$ \\
\hline$t_{a}^{0}:$ & Free-flow travel time on link $a$ \\
\hline$H_{a}:$ & Capacity of link $a$ \\
\hline$p:$ & $\begin{array}{l}\text { The number of charging facilities to be } \\
\text { located }\end{array}$ \\
\hline$q_{g}^{r s}, q_{e}^{r s}:$ & $\begin{array}{l}\text { GV and BEV travel demand between O-D } \\
\text { pair }(r, s)\end{array}$ \\
\hline$P_{k g}^{r s}, P_{k e}^{r s}:$ & $\begin{array}{l}\text { The probability that GV or BEV choose } \\
\text { path } k \text { between O-D pair }(r, s)\end{array}$ \\
\hline$\gamma_{g}, \gamma_{e}:$ & $\begin{array}{l}\text { Scale parameter of the logit model for } \\
\text { route choice of GV and BEV, respectively }\end{array}$ \\
\hline$S_{g}^{r s}, S_{e}^{r s}:$ & $\begin{array}{l}\text { The satisfaction function: the expected } \\
\text { value of the minimum perceived travel } \\
\text { time for GV and BEV travelers between } \\
\text { O-D pair }(r, s) \text { respectively }\end{array}$ \\
\hline$\xi_{k g}^{r s}, \xi_{k e}^{r s}:$ & $\begin{array}{l}\text { Random error term of perceiving } \\
\text { generalized GV and BEV path } k \text { cost } \\
\text { between O-D pair }(r, s) .\end{array}$ \\
\hline
\end{tabular}

\section{Conflicts of Interest}

The authors declare that there are no conflicts of interest regarding the publication of this paper.

\section{References}

[1] G. Haddadian, M. Khodayar, and M. Shahidehpour, "Accelerating the global adoption of electric vehicles: barriers and drivers," The Electricity Journal, vol. 28, no. 10, pp. 53-68, 2015.

[2] J. Romm, "The car and fuel of the future," Energy Policy, vol. 34, no. 17, pp. 2609-2614, 2006.

[3] Y. Nie and M. Ghamami, "A corridor-centric approach to planning electric vehicle charging infrastructure," Transportation Research Part B: Methodological, vol. 57, pp. 172-190, 2013.

[4] C. Samaras and K. Meisterling, "Life cycle assessment of greenhouse gas emissions from plug-in hybrid vehicles: Implications for policy," Environmental Science \& Technology, vol. 42, no. 9, pp. 3170-3176, 2008.

[5] L. Christensen, V. N. Anders, and A. Olsen, "Travel behaviour of potential electric vehicle drivers," in Proceedings of the European Transport Conference Glasgow, Glasgow Scotland, UK, 2010.

[6] C. Guille and G. Gross, "A conceptual framework for the vehicle-to-grid (V2G) implementation," Energy Policy, vol. 37, no. 11, pp. 4379-4390, 2009.

[7] J. A. P. Lopes, F. J. Soares, and P. M. R. Almeida, "Integration of Electric Vehicles in the Electric Power System," Proceedings of the IEEE, vol. 99, no. 1, pp. 168-183, 2011.

[8] M. Nourinejad, J. Y. J. Chow, and M. J. Roorda, "Equilibrium scheduling of vehicle-to-grid technology using activity based modelling," Transportation Research Part C: Emerging Technologies, vol. 65, pp. 79-96, 2016.

[9] M. Khan and K. M. Kockelman, "Predicting the market potential of plug-in electric vehicles using multiday GPS data," Energy Policy, vol. 46, pp. 225-233, 2012.

[10] F. He, Y. Yin, and S. Lawphongpanich, "Network equilibrium models with battery electric vehicles," Transportation Research Part B: Methodological, vol. 67, no. 3, pp. 306-319, 2014.

[11] K. Huang, P. Kanaroglou, and X. Zhang, "The design of electric vehicle charging network," Transportation Research Part D: Transport and Environment, vol. 49, pp. 1-17, 2016.

[12] B. K. Sovacool and R. F. Hirsh, "Beyond batteries: an examination of the benefits and barriers to plug-in hybrid electric vehicles (PHEVs) and a vehicle-to-grid (V2G) transition," Energy Policy, vol. 37, no. 3, pp. 1095-1103, 2009.

[13] N. Jiang, C. Xie, and S. Waller, "Path-constrained traffic assignment: model and algorithm," Transportation Research Record, vol. 2283, pp. 25-33, 2012.

[14] F. Hacker, R. Harthan, F. Matthes, and W. Zimmer, "Environmental impacts and impact on the electricity market of a large scale introduction of electric cars in Europe-Critical Review of Literature," ETC/ACC Technical Paper, vol. 4, pp. 56-90, 2009.

[15] C. Upchurch, M. Kuby, and S. Lim, "A model for location of capacitated alternative-fuel stations," Geographical Analysis, vol. 41, no. 1, pp. 127-148, 2009.

[16] I. Capar and M. Kuby, "An efficient formulation of the flow refueling location model for alternative-fuel stations," IIE Transactions, vol. 44, no. 8, pp. 622-636, 2012.

[17] M. Kuby and S. Lim, "Location of alternative-fuel stations using the flow-refueling location model and dispersion of candidate sites on arcs," Networks and Spatial Economics, vol. 7, no. 2, pp. 129-152, 2007.

[18] M. Kuby and S. Lim, "The flow-refueling location problem for alternative-fuel vehicles," Socio-Economic Planning Sciences, vol. 39, no. 2, pp. 125-145, 2005. 
[19] M. J. Hodgson, "A flow capturing location-allocation model," Geographical Analysis, vol. 22, no. 3, pp. 270-279, 1990.

[20] O. Berman, R. C. Larson, and N. Fouska, "Optimal location of discretionary service facilities," Transportation Science, vol. 26, no. 3, pp. 201-211, 1992.

[21] S. Lim and M. Kuby, "Heuristic algorithms for siting alternativefuel stations using the Flow-Refueling Location Model," European Journal of Operational Research, vol. 204, no. 1, pp. 51-61, 2010.

[22] Y.-W. Wang and C.-C. Lin, "Locating road-vehicle refueling stations," Transportation Research Part E: Logistics and Transportation Review, vol. 45, no. 5, pp. 821-829, 2009.

[23] Y.-W. Wang and C.-R. Wang, "Locating passenger vehicle refueling stations," Transportation Research Part E: Logistics and Transportation Review, vol. 46, no. 5, pp. 791-801, 2010.

[24] S. A. MirHassani and R. Ebrazi, "A flexible reformulation of the refueling station location problem," Transportation Science, vol. 47, no. 4, pp. 617-628, 2013.

[25] T. Sweda and D. Klabjan, "An agent-based decision support system for electric vehicle charging infrastructure deployment," in Proceedings of the 7th IEEE Vehicle Power and Propulsion Conference (VPPC '11), Chicago, Ill, USA, September 2011.

[26] J. Asamer, M. Reinthaler, M. Ruthmair, M. Straub, and J. Puchinger, "Optimizing charging station locations for urban taxi providers," Transportation Research Part A: Policy and Practice, vol. 85, pp. 233-246, 2016.

[27] M. Ghamami, Y. Nie, and A. Zockaie, "Planning charging infrastructure for plug-in electric vehicles in city centers," International Journal of Sustainable Transportation, vol. 10, no. 4, pp. 343-353, 2016.

[28] Z. Chen, W. Liu, and Y. Yin, "Deployment of stationary and dynamic charging infrastructure for electric vehicles along traffic corridors," Transportation Research Part C: Emerging Technologies, vol. 77, pp. 185-206, 2017.

[29] X. Xi, R. Sioshansi, and V. Marano, "Simulation-optimization model for location of a public electric vehicle charging infrastructure," Transportation Research Part D: Transport and Environment, vol. 22, pp. 60-69, 2013.

[30] J. Jung, J. Y. J. Chow, R. Jayakrishnan, and J. Y. Park, “Stochastic dynamic itinerary interception refueling location problem with queue delay for electric taxi charging stations," Transportation Research Part C: Emerging Technologies, vol. 40, pp. 123-142, 2014.

[31] J. Dong, C. Liu, and Z. Lin, "Charging infrastructure planning for promoting battery electric vehicles: an activity-based approach using multiday travel data," Transportation Research Part C: Emerging Technologies, vol. 38, pp. 44-55, 2014.

[32] F. He, D. Wu, Y. Yin, and Y. Guan, "Optimal deployment of public charging stations for plug-in hybrid electric vehicles," Transportation Research Part B: Methodological, vol. 47, pp. 87-101, 2013.

[33] R. Riemann, D. Z. W. Wang, and F. Busch, "Optimal location of wireless charging facilities for electric vehicles: flow-capturing location model with stochastic user equilibrium," Transportation Research Part C: Emerging Technologies, vol. 58, pp. 1-12, 2015.

[34] F. Wu and R. Sioshansi, "A stochastic flow-capturing model to optimize the location of fast-charging stations with uncertain electric vehicle flows," Transportation Research Part D: Transport and Environment, vol. 53, pp. 354-376, 2017.
[35] Z.-H. Zhu, Z.-Y. Gao, J.-F. Zheng, and H.-M. Du, "Charging station location problem of plug-in electric vehicles," Journal of Transport Geography, vol. 52, pp. 11-22, 2016.

[36] T.-G. Wang, C. Xie, J. Xie, and T. Waller, "Path-constrained traffic assignment: a trip chain analysis under range anxiety," Transportation Research Part C: Emerging Technologies, vol. 68, pp. 447-461, 2016.

[37] C. Xie, T. Wang, X. Pu, and A. Karoonsoontawong, "Path-constrained traffic assignment: Modeling and computing network impacts of stochastic range anxiety," Transportation Research Part B: Methodological, vol. 103, pp. 136-157, 2017.

[38] C. Xie and N. Jiang, "Relay requirement and traffic assignment of electric vehicles," Computer-Aided Civil and Infrastructure Engineering, vol. 31, no. 8, pp. 580-598, 2016.

[39] C. Xie, X. Wu, and S. Boyles, "Network equilibrium of electric vehicles with stochastic range anxiety," in Proceedings of the 17th IEEE International Conference on Intelligent Transportation Systems (ITSC '14), pp. 2505-2510, Qingdao, China, October 2014.

[40] H. Zheng, X. He, Y. Li, and S. Peeta, "Traffic equilibrium and charging facility locations for electric vehicles," Networks and Spatial Economics, vol. 17, no. 2, pp. 435-457, 2017.

[41] W. Jing, Y. Yan, I. Kim, and M. Sarvi, "Electric vehicles: a review of network modelling and future research needs," Advances in Mechanical Engineering, vol. 8, no. 1, 2016.

[42] S. C. Dafermos, "Traffic assignment problem for multiclass-user transportation networks," Transportation Science, vol. 6, no. 1, pp. 73-87, 1972.

[43] N. Jiang and C. Xie, "Computing and analyzing mixed equilibrium network flows with gasoline and electric vehicles," Computer-Aided Civil and Infrastructure Engineering, vol. 29, no. 8, pp. 626-641, 2014.

[44] S. Ryu, A. Chen, and K. Choi, "Solving the stochastic multiclass traffic assignment problem with asymmetric interactions, route overlapping, and vehicle restrictions," Journal of Advanced Transportation, vol. 50, no. 2, pp. 255-270, 2016.

[45] Y. Yang, E. Yao, Z. Yang, and R. Zhang, "Modeling the charging and route choice behavior of BEV drivers," Transportation Research Part C: Emerging Technologies, vol. 65, pp. 190-204, 2015.

[46] Q. Meng and Z. Liu, "Trial-and-error method for congestion pricing scheme under side-constrained probit-based stochastic user equilibrium conditions," Transportation, vol. 38, no. 5, pp. 819-843, 2011.

[47] W. Jing, I. Kim, M. Ramezani, and Z. Liu, "Stochastic traffic assignment of mixed electric vehicle and gasoline vehicle flow with path distance constraints," Transportation Research Procedia, vol. 21, pp. 65-78, 2017.

[48] Y. Sheffi, Urban Transportation Networks: Equilibrium Analysis with Mathematical Programming Models, Prentice-Hall, Englewood Cliffs, NJ, USA, 1985.

[49] M. Xu, Q. Meng, and K. Liu, "Network user equilibrium problems for the mixed battery electric vehicles and gasoline vehicles subject to battery swapping stations and road grade constraints," Transportation Research Part B: Methodological, vol. 99, pp. 138166, 2017.

[50] S. Nguyen and C. Dupuis, "An efficient method for computing traffic equilibria in networks with asymmetric transportation costs," Transportation Science, vol. 18, no. 2, pp. 185-202, 1984. 
[51] C. Suwansirikul, T. L. Friesz, and R. L. Tobin, "Equilibrium decomposed optimization: a heuristic for the continuous equilibrium network design problem," Transportation Science, vol.

21, no. 4, pp. 254-263, 1987. 


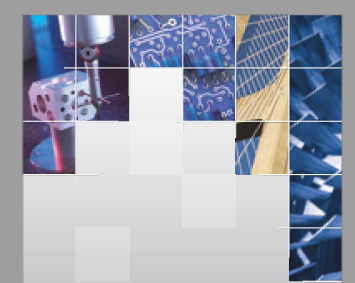

\section{Enfincering}
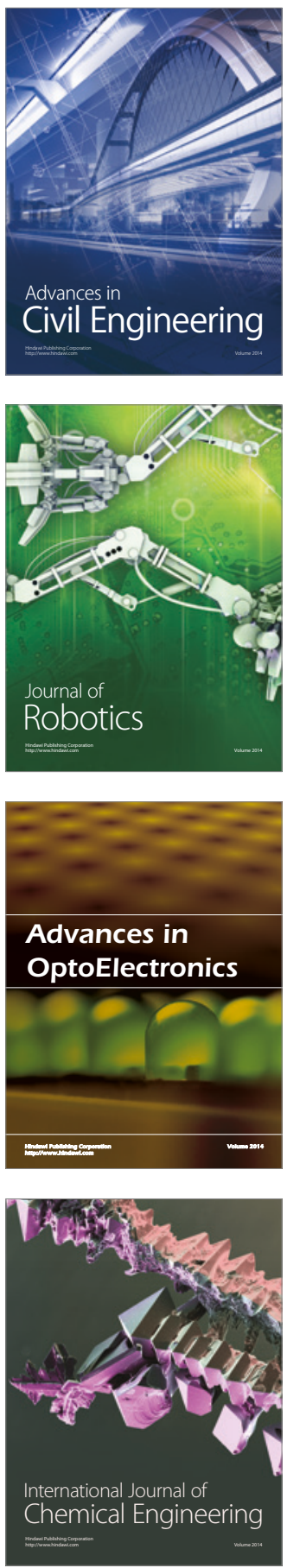

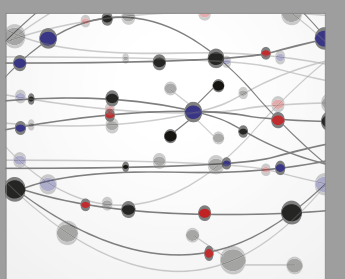

The Scientific World Journal

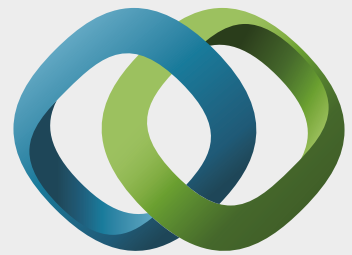

\section{Hindawi}

Submit your manuscripts at

https://www.hindawi.com
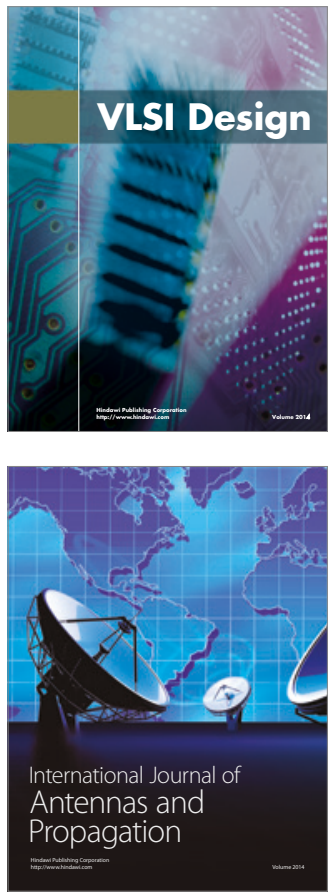

\section{Rotating}

Machinery
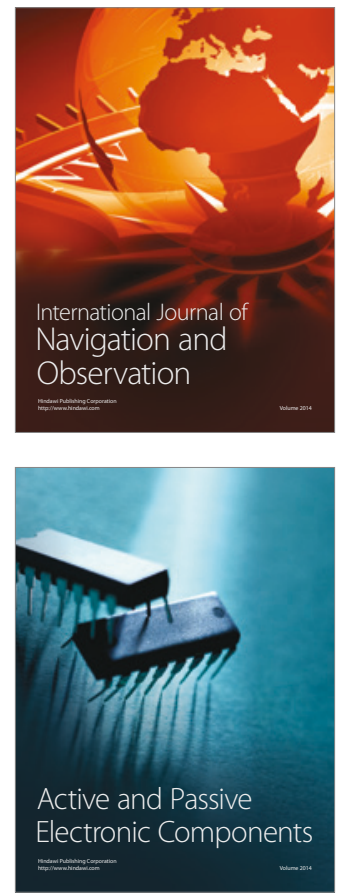
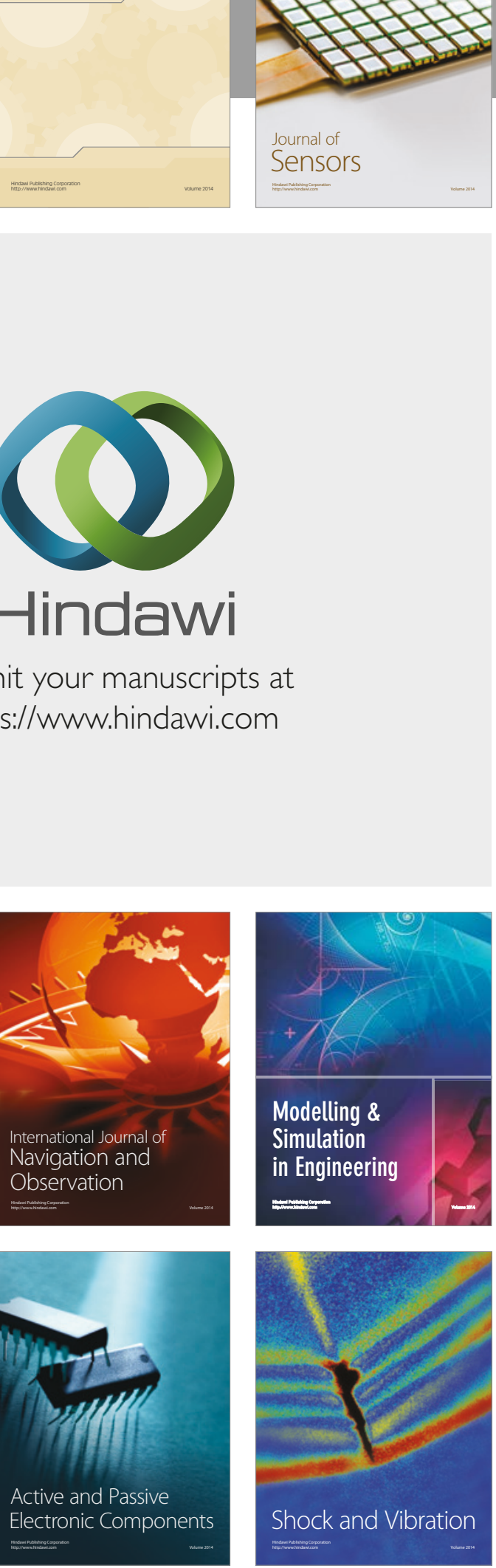
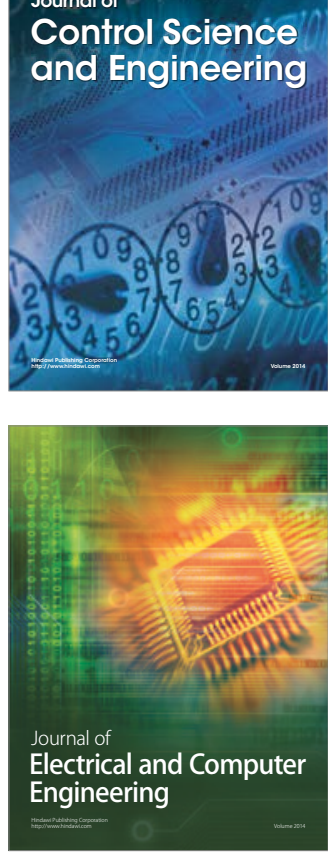

Distributed

Journal of

Control Science

and Engineering
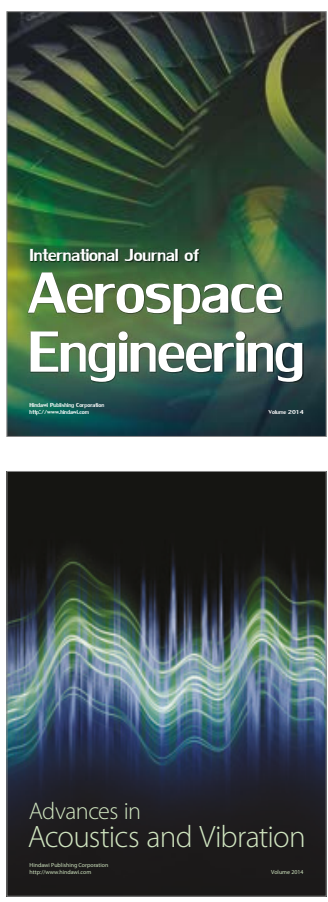

Sensor Networks 